\title{
Endobronchial and/or Endoesophageal Bronchoscopic Ultrasound-Guided Fine Needle Aspiration in the Diagnosis of Mediastinal and Hilar Diseases of Unknown Etiologies
}

\author{
Amira Abdelgalil Elkholy, ${ }^{1,}$, Ali Mohamed Abdellah ${ }^{1}$, Fawzy Abo-Elnaga El-emery ${ }^{1}$, \\ Ibrahim Salah-Eldein Ibrahim ${ }^{1}$, Ayman Abdelhamid Farghaly ${ }^{2}$, Mohamed Sayed Hantera ${ }^{1}$, \\ Ayman Mohamed El-Saka ${ }^{3}$, Dalia Ezzat Elsharawy ${ }^{1}$ \\ ${ }^{1}$ Chest Diseases Department, Faculty of Medicine, Tanta University, Tanta, Egypt \\ ${ }^{2}$ Chest Diseases Department, Kobri Elkobba Military Hospital, Medical Military Academy, Cairo, Egypt \\ ${ }^{3}$ Pathology Department, Faculty of Medicine, Tanta University, Tanta, Egypt
}

\section{Email address:}

amiraelkholy60@gmail.com(A. A. Elkholy), aabdella731@gmail.com(A. M. Abdellah), fawzy.elemairy@gmail.com (F. Abo-Elnaga El-emery), isalahi72@yahoo.com(I. Salah-Eldein I.),farghalymedkar@hotmail.com(A. A. Farghaly),dr_mohamed_hantera@hotmail.com (M. S. Hantera), aymen_saka@hotmail.com (A. M. El-Saka),dalia-elsharawy@hotmail.com (D. E. Elsharawy)

${ }^{*}$ Corresponding author

\section{To cite this article:}

Amira Abdelgalil Elkholy, Ali Mohamed Abdellah, Fawzy Abo-Elnaga El-emery, Ibrahim Salah-Eldein Ibrahim, Ayman Abdelhamid Farghaly, Mohamed Sayed Hantera, Ayman Mohamed El-Saka, Dalia Ezzat Elsharawy. Endobronchial and/or Endoesophageal Bronchoscopic Ultrasound-Guided Fine Needle Aspiration in the Diagnosis of Mediastinal and Hilar Diseases of Unknown Etiologies. International Journal of Medical Imaging. Vol. 9, No. 1, 2021, pp. 87-93. doi: 10.11648/j.ijmi.20210901.19

Received: February 11, 2021; Accepted: February 26, 2021; Published: March 4, 2021

\begin{abstract}
Introduction: Diagnosis of mediastinal and hilar lesions represents a problematic issue for long ago, not only due to various structures that occupy this space but also difficult accessibility to such lesions. Mediastinal endosonography guided FNA is emerged as a new minimally invasive modality for diagnosis of such lesions. Objectives: to evaluate the role of endobronchial and/or endoesophageal -bronchoscopic ultrasound-guided fine needle aspiration in the diagnosis of mediastinal and hilar diseases of unknown etiologies. Methods: This Prospective observational study was carried out at Tanta University Educational Hospital and Kobri Elkobba Military Hospitals; from December 2017 to December 2019 on thirty patients (19 males; 11 females, age range 17-82 years) presented with undiagnosed mediastinal and or hilar lesions. All patients were subjected to clinical examination, radiological assessment including Chest-X-Ray, CT Chest. Laboratory investigations: CBC, blood urea, serum creatinine and coagulation profile. Twenty-six patients were subjected only to endobronchial ultrasoundguided transbronchial needle aspiration (EBUS-TBNA) biopsies and four patients were subjected to endoscopic ultrasound using EBUS scope-guided fine- needle aspiration (EUS-B-FNA) after EBUS-TBNA, using Pentax Endobronchial ultrasound and a fine needle (22- Gauge). The procedure was done under local anesthesia and conscious sedation ROSE technique was applied for some cases. Results: Definitive diagnosis was reached in twenty-nine cases, achieving diagnostic yield (96.67\%). Malignant lesions were the predominant category; twenty (66.67\%), while nine (30\%) were diagnosed as benign lesions and one $(3.33 \%)$ case remained un diagnosed. Also, immunohistochemistry played an important role to reach definitive diagnosis in six cases without further sampling. About $(12 / 30 ; 40 \%)$ of patients were passed without any complications while the others recorded controlled complications without any serious events. Conclusion: EBUS and EUS-B-FNA are minimally invasive, complementary and cost effective procedures with high diagnostic yield for mediastinal and hilar lesions. Addition of immunohistochemistry raises diagnostic value of this procedure.
\end{abstract}

Keywords: Endobronchial Ultrasound, EUS-B-FNA, Mediastinal Endosonography, Mediastinal and Hilar Diseases, Immunohistochemistry 


\section{Introduction}

Mediastinal symptoms result from compression of involved structures; such trachea, esophagus and so on. [1] Divisions of mediastinum help in narrowing differential diagnosis and choosing appropriate sampling modality which is often required to confirm diagnosis and select optimal treatment. [2] Endoscopic biopsy under the guidance of ultrasonography is one of sampling techniques which can be done either through air ways (Endobronchial ultrasound; EBUS) or through the oesophagus (Endoscopic ultrasound; EUS). [3] Both EBUS-TBNA and EUS-fine needle aspiration provide a nearly complete mediastinal evaluation in a minimally invasive manner. [4]

Aim of the work was to evaluate the role of endobronchial and/or endoesophageal bronchoscopic ultrasound-guided fine needle aspiration in the diagnosis of mediastinal and hilar diseases of unknown etiologies.

\section{Patients and Methods}

\subsection{Patient Selection}

Study protocol was approved from Ethical Committee of Faculty of Medicine; Tanta University with approval code $(17 / 10 / 31806)$ and informed consents for procedure were obtained from the patients or their relatives. This Prospective observational study was carried out at bronchoscopy unit of Tanta University Educational Hospital and Kobri Elkobba Military Hospitals within 24 months; from December 2017 to December 2019. Any patient fit for bronchoscopy presented with mediastinal and or hilar lesions of unknown etiologies were included and patients who refused technique were excluded. All patients were subjected to complete clinical history taking, clinical examination and computed tomography scan of chest. Other imaging studies were requested in some cases; pelvi-abdominal US, CT brain, MRI brain, etc. for further assessment. Laboratory investigations; CBC, blood urea and serum creatinine, bleeding profile and ECG. ABG for some cases. All patients were subjected to endobronchial ultrasound-guided transbronchial needle aspiration (EBUS TBNA) and endoscopic ultrasound using EBUS scope-guided fine- needle aspiration (EUS-B-FNA) was reserved for cases in whom endobronchial approach was difficult and not feasible for sampling the lesions, after endobronchial trial using Pentax Convex Probe - Endobronchial ultrasound-1970UK, HI VISION Avius; Hitachi Company, Tokyo, Japan and a dedicated 22-Gauge Needle; ECHO-HD-22-EBUS-P of Cook Medical Company (Limerick, Ireland) for aspiration of lesions. The procedure was done under local anesthesia to nose and mouth, conscious sedation and oxygen supplementation using nasal cannula.

\subsection{Technique of the Procedure}

\subsubsection{Preparation of the Patient}

This was done through intravenous administration of sedatives (midazolam) and induction of local anesthesia to mouth and nose with lidocaine topical spray (10\%), heart rate, blood pressure and oxygen saturation monitoring were done during the procedure.

\subsubsection{Insertion of Endobronchial Ultrasound}

The bronchoscope was inserted orally then the probe passed through the vocal cords where local anesthesia with lidocaine solution $2 \%$ was injected to decrease spasm of vocal cords during passage through them. The bronchoscope was advanced through the airway until reaching desired location where local anesthesia with lidocaine solution $2 \%$ was injected again to decrease cough reflex. For ultrasound imaging, a maximum contact with the target area was done to provide a good coupling. The Doppler mode was used to identify and confirm surrounding vessels as well as the blood flow within lesions. [5]

\subsubsection{Sampling of Lesions}

Sampling of lesions through bronchial tree (EBUS pathway) was done according to Yasufuku [6] while Sampling of lesions through the esophagus (EUS-B- pathway) was done according to Bugalho et al and Jenssen et al. [7, 8]

\subsubsection{Endosonographic - Guided Fine Needle Aspiration}

Once the puncture site was decided, the needle was passed through the working channel of the bronchoscope and was secured in its place using screw movement, real-time needle aspiration performed following steps described by Sonali Sethi et al. [5]

\subsubsection{Handling of the Specimens}

When the cytopathologist was attendant, ROSE technique was applicable, first slide was stained on-site using Diff Quick stain for confirming adequacy of the specimens. When the cytopathologist was not attendant, puncture procedure was repeated for about 4 to 5 times for target lesions for obtaining more specimens.

The obtained smears were air dried, fixed in 95\% alcohol and sent for microscopic examination. Clotted materials within the needle were preserved in $10 \%$ formalin solution to be prepared as cell blocks for further examination and immunophenotyping. [9] Bronchial lavage was collected and sent for cytological and microbiological examinations. There was one case in whom sampling was not done as target lesion was distal to right main pulmonary artery, endobronchial and endoesophageal approaches were tried but transvascular approach was inevitable and was refused from the patients` relatives.

\subsection{Statistical Analysis}

Statistical analysis was conducted using SPSS version 20. Descriptive statistics for quantitative data were done using mean, standard deviation, minimum and maximum of the range while qualitative data were represented as number and percentage. Pearson's chi-squared test was applied to determine whether there is a statistically significant difference between the expected frequencies and the observed frequencies in one or more categories of a contingency table with level of significance $(\mathrm{P}$-value $\leq 0.05)$. 


\section{Results}

Regarding demographic data of the patients; this study included thirty patients with age ranged between $17-82$ years with a mean age $54.467 \pm 16.919$ ys). Nineteen cases $(63.33 \%)$ were males while eleven $(36.67 \%)$ were females. Seven cases were active smokers, eight were EX- smokers and fifteen were non-smokers. Regarding main presenting symptoms; dyspnea was the most common symptom, recorded in twenty- six $(86.67 \%)$ patients, followed by dry cough in seventeen $(56.67 \%)$, anorexia in eleven, weight loss in ten, productive cough in eight. Hemoptysis in seven, wheezy chest in seven, and low grade fever in seven, hoarseness of voice in five, chest pain in four, high grade fever in four patients, headache in four and vomiting in three patients. Dysphagia for solids in three, orthopnea in two, blurring of vision in two, constitutional symptoms (Myalgia, arthralgia and fatigue) in one, disturbed conscious level in one and paresthesia in both lower limbs in one patient. Regarding clinical signs, clubbing and pallor were the most common signs; observed in twelve patients for each. Cachexia in ten, lower limb edema in eight, fever in three, signs of SVC obstruction in one patient and papilledema in one patient while eleven patients presented with no abnormalities. CT findings were listed in Table 1.

Table 1. CT Chest findings.

\begin{tabular}{lll}
\hline & Pattern & Frequency \\
\hline $1-$ & Mediastinal lymphadenopathy with pulmonary parenchymal mass & 4 \\
$2-$ & Mediastinal lymphadenopathy with reticulonodular pattern and GGO* & 9 \\
$3-$ & Mediastinal lymphadenopathy with pleural effusion & 1 \\
$4-$ & Mediastinal lymphadenopathy with hilar mass, lung consolidation and effusion & 1 \\
$5-$ & Mediastinal lymphadenopathy with mediastinal mass, lung consolidation and pleural effusion & 1 \\
6 & Mediastinal lymphadenopathy with mediastinal mass, hilar mass lung consolidation and pleural effusion & 1 \\
7 & mediastinal mass with hilar mass & 1 \\
$8-$ & Hilar mass with emphysematous changes & 2 \\
$9-$ & Milar mass with lung consolidation and effusion & 4 \\
$10-$ & Mediastinal mass with emphysematous changes & 1 \\
$11-$ & Mediastinal mass with mediastinal lymphadenopathy and pleural effusion \\
$12-$ & & 1 \\
$13-$ & & 1 \\
\hline
\end{tabular}

* GGO: Ground glass opacities.

Regarding procedure events; Table 2, EBUS-TBNA a lone was done for twenty-six $(86.67 \%)$ patients while the other four $(13.33 \%)$ patients were subjected to EUS-B-FNA after EBUS -TBNA. The first case of the four, EUS-B-FNA was an elective additional pathway to sample the subcarinal lymph node after ensuring the adequacy of the aspirated materials from the second pass of the needle through transbronchial pathway and the patient was comfortable. In the second case, transesophageal approach was an alternative pathway to reach the lesion (which was distal to RT main pulmonary artery) before deciding failure of endosonography to reach the target lesion without transvascular puncture. The third case suffered from severe agitation and intractable cough and the fourth case had moderate to severe hypoxemia and severe agitation, both cases impeded patency of airway and necessitated to complete procedure through transesophageal pathway.

Table 2. Procedure events.

\begin{tabular}{llll}
\hline Parameter & Descriptive statistics & N & \% \\
\hline & & 26 & 86.67 \\
Type of the procedure & *EBUS-TBNA only & 4 & 13.33 \\
& *EBUS-TBNA Plus *EUS-B-FNA & $20-50$ & $37.500 \pm 8.068$ \\
Duration of procedure (Min) & Range & Mean \pm SD & $1-4$ \\
Number of lesions sampled each & Range & Mean \pm SD & $2.448 \pm 0.870$ \\
procedure & Range & $3-5$ \\
Number of passes per lesion & Mean \pm SD & $3.862 \pm 0.915$. \\
\hline
\end{tabular}

*EBUS-TBNA: Endobronchial ultrasound -guided transbronchial needle aspiration; *EUS-B-FNA: Endoscopic ultrasound using EBUS scope-guided fineneedle aspiration.

Regarding Lesions sampled through mediastinal endosonography (Table 3, Figures 1 \& 2). Lesions sampled through the esophagus were left lower paratracheal lymph nodes in two cases, subcarinal lymph node in three cases, left hilar lymph node in one case (Figure 2) and left lower paratracheal mass and left hilar mass in one case while the other lesions were sampled through EBUS-TBNA. Rapid on site evaluation (ROSE) was applicable for six (20.69\%) cases and all samples were adequate regarding quality and quantity. Immunohistochemistry (IHC) was requested for six (20.69\%) cases to reach definitive diagnosis without further sampling. It was a confirmative tool for diagnosis of two cases as small 
cell carcinoma and Hodgkin`s lymphoma, a differentiating tool in three cases, two of them diagnosed as malignant NonHodgkin`s - T- Cell lymphoma and in the third case, IHC was able to declare undifferentiated carcinoma to be as a poorly differentiated squamous cell carcinoma. The sixth case; IHC was an identical technique to change diagnosis from oat cell carcinoma with nodal metastasis to large cell lymphoma. The markers used in this study were: CD3, CD20, CD15, NSE, LCA, cytokeratin, CK5/6 and pan cytokeratin AE1/AE3, TTF-1, synaptophysin and P63.

Table 3. Mediastinal and hilar lesions sampled through mediastinal endosonography.

\begin{tabular}{llll}
\hline Parameter & Descriptive statistics $(\mathbf{n}=\mathbf{3 0})$ & & \\
\hline & & $\mathbf{N}$ & $\mathbf{\%}$ \\
\hline Mediastinal & Left lower paratracheal & 6 & 20.00 \\
masses & Right lower paratracheal & 3 & 10.00 \\
Hilar masses & Left Hilum & 6 & 20.00 \\
& Right Hilum & 3 & 10.00 \\
& Subcarinal LN (7) & 18 & 60.00 \\
Lymph nodes & Right hilar LN (10R) & 12 & 40.00 \\
stations & Left hilar LN (10L) & 11 & 36.67 \\
& Right lower paratracheal LN *(4R) & 9 & 30.00 \\
& Left lower paratracheal LN* (4L) & 7 & 23.3 \\
\hline
\end{tabular}

*LN: lymph node
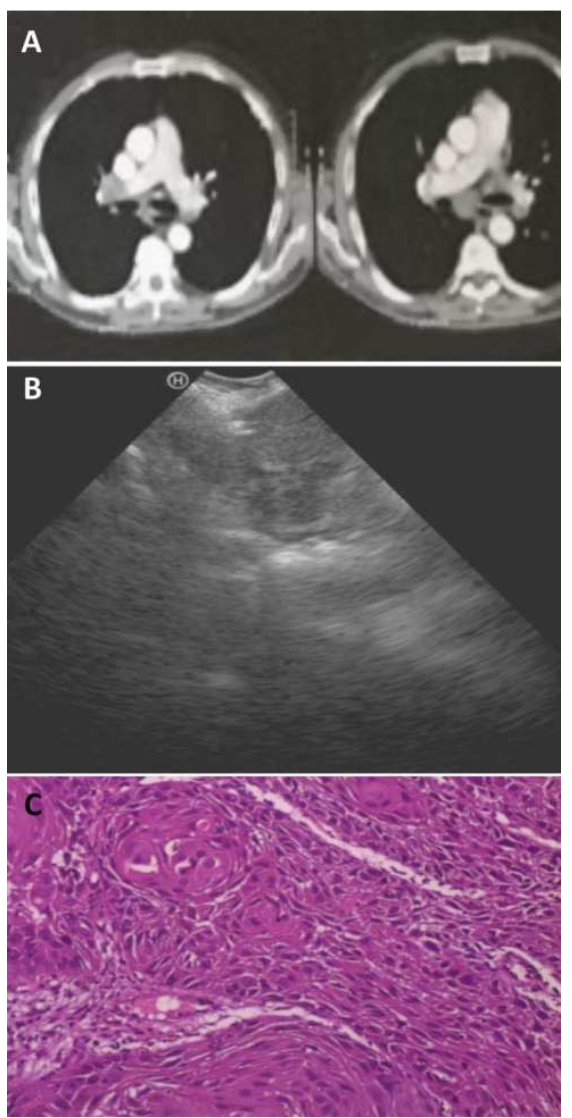

Figure 1. A): CT Chest with contrast, mediastinal window showed mediastinal lymphadenopathy at hilar and subcarinal regions. B): Ultrasound image showed EBUS-guided fine needle sampling sub-carinal lymph node. C): Microscopic picture showed malignant sheets and cell nests exhibiting squamous cells with moderate degree of pleomorphism and high $N / C$ ratio with central keratin formation, consistent with Squamous cell lung carcinoma $(H \& E \times 100)$.

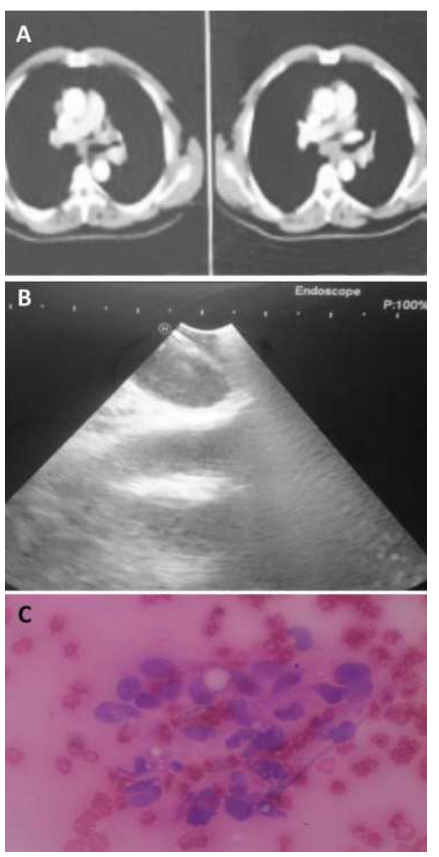

Figure 2. A): CT Chest with contrast, mediastinal window showed mediastinal lymphadenopathy at hilar and subcarinal regions. B): Ultrasound image showed EUS-B-guided fine needle sampling left hilar lymph node. C): Microscopic picture showed non caseating inflammatory granuloma, which is pathologically consistent with sarcoidosis (H\&E $\times 400)$.

Regarding nature of aspirated specimens; fluid aspirates for smear slide were obtained for twenty-nine (96.67\%) cases for cytological examination and number of slides for each patient ranged between 5-29 slides with a mean \pm SD, $14.552 \pm 6.780$ slides. Also, Clotted materials for cell blocks were obtained for twenty (66.67\%) cases for doing IHC when needed. Longest diameter of Clotted materials ranged between 0.3-1.5 cm with a mean $\pm \mathrm{SD}, 0.650 \pm 0.319 \mathrm{~cm}$. Bronchial lavage was obtained for twenty $(66.67 \%)$ cases for cytological examination and doing special stain (ZeihlNeelsen stain) when requested. The amount of lavage ranged between 20-100 cc with a mean $\pm \mathrm{SD}, 62.250 \pm 23.310 \mathrm{cc}$.

Regarding complications recorded; twelve patients (40\%) were passed without any complications, while eight cases (26.67\%) suffered from hypoxemia during the procedure which was mild to moderate, transient and rapidly corrected with rising oxygen flow except one case; hypoxemia was moderate to severe and necessitated to shift to esophageal pathway. Agitation was recorded in seven cases which was ameliorated with increase dose of sedatives. Cough and bleeding were recorded in six cases for each. Cough was tolerated except one case; cough was severe, intractable and necessitated to shift to esophageal pathway. Bleeding was mild and controlled with suction and lavage using cold saline. Post bronchoscopic bronchospasm was the least complication recorded only in one case $(3.33 \%)$ and rapidly relieved using bronchodilator nebulizer.

Regarding definitive diagnosis, collected in Table 4. It was noticed that there was a statistically significant relation between sex, smoking and incidence of malignancy with a P- 
value (0.001) and (0.032) respectively. On the other hand, there was no significant relationship with age. Distribution of histopathology regarding age groups, sex and smoking status collected in Table 5.

Table 4. Definitive Diagnosis of the patients.

\begin{tabular}{|c|c|c|c|}
\hline \multicolumn{4}{|c|}{ Definitive Diagnosis } \\
\hline \multicolumn{2}{|c|}{ Diagnostic Categories } & $\mathbf{N}$ & $\%$ \\
\hline No diagnosis & No diagnosis & 1 & 3.33 \\
\hline \multirow{2}{*}{ Benign lesions } & Sarcoidosis & 8 & 26.67 \\
\hline & Chronic non- specific Inflammation & 1 & 3.33 \\
\hline \multirow{8}{*}{ Malignant lesions } & NSCLC- Large cell carcinoma & 4 & 13.33 \\
\hline & Small cell lung carcinoma & 4 & 13.33 \\
\hline & *NSCLC- Poorly differentiated squamous cell carcinoma & 5 & 16.67 \\
\hline & *NSCLC- Poorly differentiated adenocarcinoma & 2 & 6.67 \\
\hline & Lymphoma & & \\
\hline & Large cell lymphoma & 2 & 6.67 \\
\hline & Hodgkin`s lymphoma & 1 & 3.33 \\
\hline & Non-Hodgkin`s T- cell lymphoma & 2 & 6.67 \\
\hline
\end{tabular}

*NSCLC: Non-small cell lung cancer.

Table 5. Distribution of histopathology regarding age groups, sex and smoking status.

\begin{tabular}{|c|c|c|c|c|c|c|c|c|c|c|}
\hline \multirow{3}{*}{ Age group (Ys) } & \multicolumn{10}{|c|}{ Number of patients } \\
\hline & \multicolumn{7}{|c|}{ Malignant lesions } & \multicolumn{2}{|c|}{ Benign lesions } & \multirow{2}{*}{ Total } \\
\hline & $* \mathbf{H L}$ & *NHL & ${ }^{*}$ L.C.L & ${ }^{*}$ SQ.C.C & *S.C.C & ${ }^{*}$ L.C.C & Adenocarcinoma & Sarcoidosis & Chronic *NSI & \\
\hline $17-30$ & 1 & 2 & & & & 1 & & & & 4 \\
\hline $31-40$ & & & & & & & & 1 & & 1 \\
\hline $41-50$ & & & & & 1 & & 1 & 2 & 1 & 5 \\
\hline $51-60$ & & & 1 & 2 & 3 & 1 & & 4 & & 11 \\
\hline $61-70$ & & & & 1 & & 1 & 1 & 1 & & 4 \\
\hline Male & & 2 & 2 & 5 & 4 & 2 & 2 & 1 & & 18 \\
\hline Female & 1 & & & & & 2 & & 7 & 1 & 11 \\
\hline Active smoker & & 1 & 1 & 2 & 3 & & & & & 7 \\
\hline EX smoker & & & 1 & 3 & 1 & & 1 & 1 & & 7 \\
\hline Non smoker & 1 & 1 & & & & 4 & 1 & 7 & 1 & 15 \\
\hline
\end{tabular}

*HL: Hodgkin`s lymphoma *NHL: Non-Hodgkin`s lymphoma *LCL: Large cell lymphoma

*SQ.C.C: squamous cell carcinoma *S.C.C: Small cell carcinoma *L.C.C: Large cell carcinoma.

*Chronic NSI: Chronic non- specific Inflammation.

\section{Discussion}

For many years, surgical biopsies took the upper hand for diagnosis of mediastinal and hilar lesions which are beyond bronchoscopic field. Nowadays, with evolution of ultrasonography and its integration with endoscopy and appearance of EUS and EBUS enable endoscopists and bronchoscopists for sampling of these lesions. Mediastinal endosonography (EBUS-TBNA and EUS-FNA or EUS-BFNA) has been recommended in recent guidelines as an initial test of choice for NSCLC staging over surgical one. EUS-B-FNA has been introduced recently in pulmonology intervention using an ultrasound bronchoscope through the esophagus. [5] Helalah et al., 2019 [10] were in agreement with our study as dyspnea was the predominant symptoms. On the other hand, Hantera et al., 2019 [11] and Pulasani et al., 2015 [12] also reported that dry cough was the predominant symptoms followed by breathlessness. While Dixit et al., 2017 [13] reported that superior vena cava obstruction was the most common sign, this study recorded SVC obstruction only in one case. Regarding radiological patterns, mediastinal and hilar masses and mediastinal lymphadenopathies were the targets for sampling using
EBUS and EUS-B-FNA, all were sampled except one case; transvascular approach was inevitable and we had a fear of serious complications as the patient was 82 ys old and his relatives also refused this approach.

In agreement with this study Zhang et al., 2013 [14] who reported that both techniques are complementary methods for reaching mediastinal and hilar nodal stations. Also, Tournoy et al., 2012 [15] reported that EUS is better tolerated than EBUS as no coughing or dyspnea so, it can be applied to (station 4 L) and (station 7). Not only but also, Araya et al., 2013 [16] reported that EUS-B-FNA is a feasible alternative method for lesions in patients with persistent cough or to poor physical condition. Also, Vilmann et al., 2005 [17] found that accuracy of combined EUS-FNA and EBUSTBNA for the diagnosis of mediastinal cancer was $100 \%$ and both techniques are complementary to each other.

Regarding unsampled case, in agreement with this point Botana-Rial et al., 2012 [18] reported the first case of an intramural hematoma of pulmonary artery with a secondary hemopneumomediastinum when the needle was accidentally passed into pulmonary artery. Also, LeBlanc et al., 2004 [19] concluded that five lymph nodes passes are required to reach a definite diagnosis so, transvascular approach is of low 
sensitivity. On the other hand, Kazakov et al., 2017 [20] concluded that EBUS and EUS-guided transvascular biopsy of mediastinal, hilar and lung lesions was not associated with significant complications but careful selection of the patients was needed. In accordance with our study, Dhooria et al., 2019 [21] reported similar events, bleeding and excessive cough so, EBUS-TBNA replaced by EUS-B-FNA.

Regarding the duration of the procedure and number of passes per lesion, this study showed agreement with Helalah et al., 2019 [10] and Abu-Hijleh et al., 2013 [22], not only but also, Lee et al., 2008 [23] recommended at least 3 aspirates at each LN station for lung cancer staging. Regarding lesions sampled through mediastinal endosonography; there was an agreement with Helalah et al., 2019 [10], Hantera et al., 2019 [11] and Dhooria et al., 2019 [21]; the most frequent LN station sampled was subcarinal.

Regarding Rapid on Site Evaluation technique; it was adequate regarding quality and quantity of the specimens. On the other hand, Rose technique did not affect the result of fine needle aspiration rather than reduction in number of passes per lesion and number of lesions targeted as well as duration of procedure. In agreement with our study Dhooria et al., 2019 [21] found that ROSE did not affect the diagnostic yield and it was performed in only a small number. Also, Wong et al., 2014 [24] found that ROSE led to a significant reduction in mean number of lesions sampled and needle punctures. On the other hand, Bediwy et al., 2017 [9] found that ROSE improves diagnostic accuracy of EBUS-TBNA and provides sufficient specimens for subsequent immunocytochemistry.

Regarding immunohistochemistry, it showed a valuable role for reaching final diagnosis, in accordance with our study Wong et al., 2014 [24] reported that immunohistochemistry was not performed in all cases where malignancy was confirmed in cell block. But, in some cases, cell block immunohistochemistry provided additional data other than lung carcinoma immunophenotype. Liu et al., 2017 [25] reported that IHC studies are very helpful in the differential diagnosis of malignancy. Regarding nature of aspirated specimens; Coley et al., 2015 [26] reported that the FNA cell blocks and direct FNA smears show comparable results with core needle biopsy samples for molecular testing.

Also, Van Zante and Ljung, 2016 [27] informed that correct technique using FNAs with ROSE is superior to that of a core needle biopsy samples, also, Mehta et al., 2015 [28] found that most of LNs sampled during EBUS-FNA with negative and unsatisfactory results were likely to be truly negative on follow-up. Regarding complications reported in our study, Von Bartheld et al., 2014 [29] reported in their review for 16,181 patients, there were (23) serious adverse events, (35) minor events with no mortality. Regarding definitive diagnosis, Dixit et al., 2017 [13] also found that malignancy was the most common category $(67 \%)$ and metastatic carcinoma was the predominant pathology. Also, Dhooria et al., 2019 [21] found that EBUS-TBNA may be an initial procedure for mediastinal lymphoma diagnosis and its subtyping. Our study showed accordance with their study specially in the point of lymphoma diagnosis and use of IHC in its subtyping. Also, Yasufuku et al [3] found that EBUS-TBNA was diagnostic in 131 of 140 patients $(93.6 \%)$; malignant in $(87.5 \%)$ and benign in $(96.0 \%)$. On the other hand, Annema et al., 2008 [30] recommended surgery for suspicious lymph nodes on CT or PET with negative endosonography.

\section{Conclusion}

Performing EBUS and EUS-B FNA at the same session by the same apparatus and operator is a time saving, cost effective and more tolerable technique with better diagnostic yield. Addition of immunohistochemistry to fine-needle aspirates improves diagnostic accuracy and subtyping of lung cancer, as well as, it spares the patients for further sampling either by the same procedure or referral to more invasive surgical modalities especially in diagnosis of lymphoma and its subtyping which usually requires excision biopsy for accurate diagnosis.

\section{Conflict of Interest}

The authors declare that they have no conflict of interest.

\section{Disclosures and Funding Sources}

NIL.

\section{Acknowledgements}

The authors would like to thank Mr. Ahmed Samir Ryad for the technical assistance in the manuscript.

\section{References}

[1] Wright CD and Mathisen DJ. (2001): Mediastinal tumors, diagnosis and treatment. World J Surg.; 25 (2): 204-9.

[2] Whitten CR, Khan S, Munneke GJ, Grubnic S. (2007): A Diagnostic Approach to Mediastinal Abnormalities. RadioGraphics.; 27: 657-671.

[3] Yasufuku K, Nakajima T, Fujiwara T, Yoshino I, Keshavjee S. (2011): Utility of endobronchial ultrasound-guided transbronchial needle aspiration in the diagnosis of mediastinal masses of unknown etiology. Ann Thorac Surg.; 9 (3): $831-836$.

[4] VanderLaan PA, Wang HH, Majid A, Folch E. (2014): Endobronchial Ultrasound-Guided Transbronchial Needle Aspiration (EBUS-TBNA): An Overview and Update for the Cytopathologist. American Cancer Society.; 122 (8): 561-76.

[5] Sonali Sethi and Joseph Cicenia. (2013): EBUS-TBNA Bronchoscopy. In: Interventional Bronchoscopy, A clinical guide Respiratory Medicine (10 $0^{\text {th }}$ ed). EDS: Mehta AC and Jain p "Humana Press, "Springer"; 5: 85-106.

[6] Yasufuku K. (2009): EBUS-TBNA Bronchoscopy. In: Endobronchial Ultrasound an Atlas and Practical Guide. EDS: Ernst A and Herth FJF "Springer"; 8: 119-144. 
[7] Bugalho A, De Santis M, Szlubowski A, Rozman A, Eberhardt R. (2018): Trans-esophageal endobronchial ultrasound-guided needle aspiration (EUS-B-NA): A road map for the chest physician. Pulmonology.; 24 (1): 32-41.

[8] Jenssen C, Annema JT, Clementsen P, Cui XW, Borst MM, Dietrich CF. (2015): Ultrasound techniques in the evaluation of the mediastinum, part 2: mediastinal lymph node anatomy and diagnostic reach of ultrasound techniques, clinical work up of neoplastic and inflammatory mediastinal lymphadenopathy using ultrasound techniques and how to learn mediastinal endosonography. J Thorac Dis.; 7 (10): 439458.

[9] Bediwy AS, Zamzam Kh, Hantira M, El-Sharawy D, ElSaqa A, Zamzam Y. (2017): The value of rapid on-site evaluation during endobronchial ultrasound-guided transbronchial needle aspiration in the diagnosis of mediastinal lesions. The Egyptian Journal of Bronchology.; 11 (4): 336-341.

[10] Helalah LA, Madkour AM, A. Elfattah NM, Mohammed RM, Farghaly A, Fawzy RA, et al. (2019): The role of convex probe endobronchial ultrasound-guided transbronchial needle aspiration in the diagnosis of hilar and mediastinal lesions. The Egyptian journal of bronchology.; 13 (5): 644-653.

[11] Hantera MSA, Abdalla HAH and Ibrahim MA. (2019): Mediastinal lesions, spectrum, and modalities of diagnosis: a retrospective multicenter-based experience. The Egyptian journal of bronchology.; 13 (3): 370-376.

[12] Pulasani K, Narayanaswamy I and Ramprakash HV. (2015): Evaluation of Mediastinal Mass Lesions Using Multi-detector Row Computed Tomography and Correlation with Histopathological Diagnosis. International Journal of Scientific Study.; 3 (6): 156-163.

[13] Dixit R, Shah NS, Goyal M, Patil CB, Panjabi M, Gupta RC, et al. (2017): Diagnostic evaluation of mediastinal lesions. Lung India.; 34 (4): 341-348.

[14] Zhang R, Ying K, Shi L, Zhang L, Zhou L. (2013): Combined endobronchial and endoscopic ultrasound-guided fine needle aspiration for mediastinal lymph node staging of lung cancer: a metaanalysis. Eur J Cancer.; 49 (8): 1860-7.

[15] Tournoy KG, Keller SM and Annema JT. (2012): Mediastinal staging of lung cancer: novel concepts. Lancet Oncol.; 13 (5): 221-229.

[16] Araya T, Demura Y, Kasahara K, Matsuoka H, Yamamura K, Nishitsuji M, et al. (2013): Usefulness of Transeso-phageal Bronchoscopic Ultrasound-guided Fine-Needle Aspiration in the Pathologic and Molecular Diagnosis of Lung Cancer Lesions Adjacent to the Esophagus. $J$ Bronchol Intervent Pulmonol.; 20, (2): 121-126.

[17] Vilmann P, Krasnik M, Larsen SS, Jacobsen GK, Clementsen P. (2005): Transesophageal endoscopic ultrasound-guided fine-needle aspiration (EUS-FNA) and endobronchial ultrasound-guided transbronchial needle aspiration (EBUSTBNA) biopsy: a combined approach in the evaluation of mediastinal lesions. Endoscopy.; 37 (9): 833-9.

[18] Botana-Rial M, Núñez-Delgado M, Pallarés-Sanmartín A, Leiro-Fernández V, Represas Represas C, González Silva AI, et al. (2012): Intramural hematoma of the pulmonary artery and hemop-neumomediastinum after endobronchial ultrasound-guided transbronchial needle aspiration. Respiration.; 83 (4): 353-356.
[19] LeBlanc JK, Ciaccia D, Al Assi MT, McGrath K, Imperiale T, Tao LC, Vallery S, et al. (2004): Optimal number of EUSguided fine needle passes needed to obtain a correct diagnosis. Gastrointest Endosc.; 59 (4): 475-481.

[20] Kazakov J, Hegde P, Tahiri M, Thiffault V, Ferraro P, Liberman M. (2017): Endobronchial and Endoscopic Ultrasound -Guided Transvascular Biopsy of Mediastinal, Hilar, and Lung lesions. Ann Thorac Surg.; 103 (3): 951-955.

[21] Dhooria S, Mehta RM, Madan K, Vishwanath G, Sehgal IS, Chhajed PN, et al. (2019): A Multicenter Study on the Utility of EBUS-TBNA and EUS-B-FNA in the Diagnosis of Mediastinal Lymphoma. J Bronchology Interv Pulmonol.; 26 (3): 199-209.

[22] Abu-Hijleh M, El-Sameed Y, Eldridge K, Vadia E, Chiu H, Dreyfuss Z, et al. (2013): Linear Probe Endobronchial Ultrasound Bronchoscopy with Guided Transbronchial Needle Aspiration (EBUS-TBNA) in the Evaluation of Mediastinal and Hilar Pathology: Introducing the Procedure to a Teaching Institution. Lung.; 191 (1): 109-15.

[23] Lee HS, Lee GK, Lee HS, Kim MS, Lee JM, Kim HY, et al. (2008): Real-time endobronchial ultrasound-guided transbronchial needle aspiration in mediastinal staging of nonsmall cell lung cancer: how many aspirations per target lymph node station? Chest.; 134 (2): 368-374.

[24] Wong RWM, Thai A, Khor YH, Ireland-Jenkin K, Lanteri CJ, Jennings BR. (2014): The Utility of Rapid On-Site evaluation on endobronchial ultrasound guided transbronchial needle aspiration: does it make a difference?. Journal of Respiratory Medicine.; 2014: 1-5.

[25] Liu A, Qian L, Zhong Y, Lu X, Zhao Y. (2017): Endobronchial ultrasound guided transbronchial needle aspiration combining with immunohistochemistry and genotype in lung cancer, A single - center, 55 cases retrospective study. Annals of Medicine and Surgery.; 23: 1-7.

[26] Coley SM, Crapanzano JP and Saqi A. (2015): FNA, core biopsy, or both for the diagnosis of lung carcinoma: obtaining sufficient tissue for a specific diagnosis and molecular testing. Cancer Cytopathol.; 123 (5): 318-326.

[27] Van Zante A and Ljung BM. (2016): Fine-needle aspiration versus core needle biopsy: reconsidering the evidence of superiority. Cancer.; 124 (12): 853-856.

[28] Mehta HJ, Tanner NT, Silvestri G, Simkovich SM, Shamblin C, Shaftman SR, et al. (2015): Outcome of patients with negative and unsatisfactory cytologic specimens obtained by endobronchial ultrasoundguided transbronchial fine-needle aspiration of mediastinal lymph nodes. Cancer Cytopathol.; 123 (2): 92-97.

[29] Von Bartheld MB, van Breda A and Annema JT. (2014): Complication rate of Endosonography (Endobronchial and Endoscopic Ultrasound): A Systematic Review. Respiration.; 87 (4): 343-351.

[30] Schipper P and Schoolfield M. (2008): Minimally invasive staging of $\mathrm{N} 2$ disease: endobronchial ultrasound /transesophageal endoscopic ultrasound, mediastinoscopy and thoracoscopy. Thorac Surg Clin.; 18 (4): 363-79. 